Minimal and maximal plateau lengths in Motzkin paths 


\title{
The Size of the rth Smallest Component in Decomposable Structures with a Restricted Pattern
}

\author{
Li Dong ${ }^{1}$ and Zhicheng Gao ${ }^{12}$ and Daniel Panario ${ }^{1}$ \\ ${ }^{1}$ Carleton University, Mathematics and Statistics, 1125 Colonel By Drive, Ottawa ON, K1S 5B6, Canada \\ ${ }^{2}$ Center for Combinatorics, LPMC, Nankai University, Tianjin, 300071, China
}

\begin{abstract}
In our previous work (2), we derived an asymptotic expression for the probability that a random decomposable combinatorial structure of size $n$ in the exp-log class has a given restricted pattern. In this paper, under similar conditions, we provide the probability that a random decomposable combinatorial structure has a given restricted pattern and the size of its $r$ th smallest component is bigger than $k$, for $r, k$ given integers. Our studies apply to labeled and unlabeled structures. We also give several concrete examples.
\end{abstract}

Keywords: decomposable combinatorial structures; restricted pattern; exp-log class; singularity analysis; $r$ th smallest component.

\section{Introduction}

Let $\mathcal{C}$ be a class of combinatorial structures. We call $\mathcal{F}$ a class of decomposable combinatorial structures over $\mathcal{C}$ if each element of $\mathcal{F}$ can be uniquely decomposed into a multiset of elements of $\mathcal{C}$. The elements of $\mathcal{C}$ are called the components.

Traditionally, combinatorial structures are divided into two categories depending whether structures under consideration are labeled or unlabeled. Let $C_{k}$ be the number of elements of size $k$ in $\mathcal{C}$. For labeled structures, the (exponential) generating function of $\mathcal{C}$ is $C(z)=\sum_{k \geq 0} C_{k} z^{k} / k$ !. An element of $\mathcal{F}$ is formed by taking a multiset of labeled elements of $\mathcal{C}$ and performing all consistent relabellings. It is well known (for example, see (5) that the exponential generating function of $\mathcal{F}$ is given by

$$
F(z)=\exp (C(z)) .
$$

For unlabeled structures, the (ordinary) generating function of $\mathcal{C}$ is $C(z)=\sum_{k>0} C_{k} z^{k}$. Since each element of $\mathcal{F}$ is obtained by taking a multiset of elements of $\mathcal{C}$, the ordinary generating function of $\mathcal{F}$ is given by (see (5) )

$$
F(z)=\exp \left(C(z)+\frac{C\left(z^{2}\right)}{2}+\frac{C\left(z^{3}\right)}{3}+\cdots\right) .
$$

It is well known that the asymptotic growth of the coefficients of a generating function is determined by the behavior of its singularities. In (6), Flajolet and Soria systematically studied 1365-8050 (c) 2007 Discrete Mathematics and Theoretical Computer Science (DMTCS), Nancy, France 
the distribution of the number of components in a large class of decomposable combinatorial structures, which they called the exp-log class. Roughly speaking, a decomposable combinatorial structure is in the exp-log class if the generating function $C(z)$ has a unique singularity $\rho$ on its circle of convergence and $C(z)$ behaves like $\log (1-z / \rho)$ around $z=\rho$ (See Section 2 for a precise definition). Flajolet and Soria showed that the distribution of the number of components in a random decomposable structure of size $n$ is asymptotically normal with mean and variance about $\log n$.

In (2), we studied decomposable combinatorial structures in the exp-log class with a given restricted pattern, where by a restricted pattern, we mean that the number of components of certain sizes are specified in advance. Asymptotic expressions were derived for the probability that a random decomposable structure of size $n$ in the exp-log class has a given restricted pattern.

In this paper, we shall continue our work in (2) to study the probability of a random decomposable structure which has a given restricted pattern and a restricted size on its $r$ th smallest component. Throughout this paper, a random structure is chosen uniformly from a set under consideration. Panario and Richmond (14) have studied the equivalent subject when there is no restricted pattern. The similar problem of the distribution of the size of the $r$ th largest component has been studied by Gourdon (9) in his Ph.D thesis. In addition, many studies of restricted patterns for particular structures have been done, such as the distribution of cycles in permutations $(7,8)$ and the distribution of irreducible factors in polynomials over finite fields (1; 10, 12). Among those studies, singularity analysis of generating function brought in by Flajolet and Odlyzko (4) already became a powerful tool for extracting relevant asymptotic information.

The paper is organized as follows. Section 2 starts with the definition of the exp-log class which covers a large number of decomposable combinatorial structures. We refer to some classical tools from analytic combinatorics: generating functions and singularity analysis. We also review two theorems (Theorems 1 and 2 of our previous work (2) and prove two extended theorems (Theorem 3 and 4 from the previous paper (2) which are applied to prove the theorems in this paper. In Section 3 , the main theorems of this paper are given (Theorem 5 for the labeled case and Theorem 6 for the unlabeled case). Because the estimates provided by the main theorems depend on the given restricted pattern, we can simplify our results greatly by setting stricter conditions on the pattern. The simplified results are presented in Corollaries 1, 2, 3, and4. In Section 4, we discuss the first moment of the size of the $r$ th smallest component for a decomposable structure in the exp-log class with a restricted pattern (Theorem/7 and Corollary 5). Section 5 exemplifies our results for some decomposable combinatorial structures, including labeled and unlabeled cases. Finally, some problems for further research are introduced in Section 6.

\section{Background and Previous Results}

In this paper, we shall restrict our attention to decomposable combinatorial structures in the exp-log class. As in (2), we use the following definition which is equivalent to that introduced in (6), and is more convenient for our purpose.

Definition 1 Let $\triangle(\nu, \theta)$ be the region $|z| \leq 1+\nu$ minus the region $|\arg (z-1)| \leq \theta$, with $\nu>0$ 
and $0<\theta<\pi / 2$. We say that $C(z)$ is of logarithmic type with multiplicity constant $a>0$ if

$$
C(\rho z)=a \log \left(\frac{1}{1-z}\right)+R(z)
$$

where the positive constant $\rho$ is the radius of convergence of $C(z), R(z)$ is analytic in $\triangle(\nu, \theta)$, and as $z \rightarrow 1$ in $\triangle(\nu, \theta), R(z)=K+O\left((1-z)^{\alpha}\right)$ with $0<\alpha<1$ and $K$ a complex constant. If the component generating function $C(z)$ is of logarithmic type, we say that the corresponding structure $\mathcal{F}$ is in the exp-log class.

Applying Flajolet-Odlyzko's transfer theorem (4), we obtain

$$
\left[z^{n}\right] C(z)=\frac{1}{\rho^{n}} \frac{a}{n}\left(1+O\left(n^{-\alpha}\right)\right),
$$

where $\left[z^{n}\right] C(z)$ denotes the coefficient of $z^{n}$ in $C(z)$.

In the labeled case, it follows from (1) and Flajolet-Odlyzko's transfer theorem that

$$
\left[z^{n}\right] F(z)=\frac{1}{\rho^{n}} \frac{e^{K}}{\Gamma(a)} n^{a-1}\left(1+O\left(n^{-\alpha}\right)\right) .
$$

In the unlabeled case, we require the singular point $0<\rho<1$ such that the radius of convergence of

$$
\frac{C\left(z^{2}\right)}{2}+\frac{C\left(z^{3}\right)}{3}+\cdots
$$

is bigger than $\rho$. Let

$$
r_{0}=\frac{C\left(\rho^{2}\right)}{2}+\frac{C\left(\rho^{3}\right)}{3}+\cdots .
$$

Then the transfer theorem and (1) entails

$$
\left[z^{n}\right] F(z)=\frac{1}{\rho^{n}} \frac{e^{K+r_{0}}}{\Gamma(a)} n^{a-1}\left(1+O\left(n^{-\alpha}\right)\right) .
$$

Now we recall from (2) the definition of restricted pattern.

Definition $2 A$ restricted pattern for a structure is defined as a mapping $S: J \mapsto N$, where $J$ is the set of the components' sizes specified in advance and $N$ is the set of nonnegative integers. Then $S(j)$ is the number of components of size $j$ in the given restricted pattern $S$.

To illustrate the above definition, consider permutations whose components are cycles. If $J$ is the empty set, then there is no restricted pattern and we are dealing with all the permutations. If the restricted pattern is $S(j)=0$ for $1 \leq j \leq k$, then we are dealing with permutations whose cycle lengths are all greater than $k$.

In the rest of the paper we use the following notations from (2) (we recall that $C_{j}$ is the number of components of size $j$ ):

- $|A|$ denotes the number of elements in a set $A$; 
- $N_{k}=\{1,2, \ldots, k\}$;

- Given a set $A$ of positive integers, $h(z ; A)=\prod_{i \in A}\left(1-z^{i}\right)^{C_{i}}$;

- $d(k)=\sum_{j \in J \cap N_{k}} S(j)$

- $C_{L}(z ; J)=\sum_{j \in J} C_{j} \frac{z^{j}}{j !}$ and $\mathscr{S}_{L}(J)=\prod_{j \in J} \frac{C_{j}^{S(j)}}{(j !)^{S(j)} S(j) !} ;$

- $C_{U}(z ; J)=\sum_{j \in J} C_{j} z^{j}$ and $\mathscr{S}_{U}(J)=\prod_{j \in J}\left(\underset{S(j)}{C_{j}+S(j)-1}\right)$;

- $m=n-\sum_{j \in J} j S(j)$ denotes the size of the unrestricted part of the structure of size $n$ (one may think of $m$ as the degree of freedom of a structure of size $n$ with a given restricted pattern $S$ ).

In the previous paper (2), we have obtained the probability that a random decomposable combinatorial structure with size $n$ has a given pattern when the sizes of the components in the pattern are negligible compared to $n$. In the following (Theorems 3 and 44, we extend the patterns in our theorems to include large size components. As it can be seen, we obtain the same probability estimates as in our previous work.

Let us first review two theorems from (2) which give the generating functions of the decomposable combinatorial structures with given restricted patterns.

Theorem 1 Let $S: J \mapsto N$ be a given restricted pattern. The exponential generating function of labeled structures with size $n$ and restricted pattern $S$ is

$$
L(z ; S)=\exp \left(C_{L}(z)-C_{L}(z ; J)\right) \prod_{j \in J} \frac{C_{j}^{S(j)} z^{j S(j)}}{(j !)^{S(j)} S(j) !} .
$$

Theorem 2 Let $S: J \mapsto N$ be a given restricted pattern. The generating function of unlabeled structures with size $n$ and restricted pattern $S$ is

$$
U(z ; S)=\left(\prod_{j \in J}\left(1-z^{j}\right)^{C_{j}}\left(\begin{array}{c}
C_{j}+S(j)-1 \\
S(j)
\end{array}\right) z^{j S(j)}\right) \exp \left(C_{U}(z)+\frac{C_{U}\left(z^{2}\right)}{2}+\cdots\right) .
$$

Then, we extend two theorems from our previous paper (2) for labeled and unlabeled structures. The extensions below cover large size components in the restricted pattern.

Theorem 3 Let $P_{L}(S, n)$ be the probability that a random decomposable labeled structure with size $n$ has a restricted pattern $S$. Let $J^{(1)}=\{j \in J: j \leq m\}$ and $J^{(2)}=\{j \in J: j>m\}$. Suppose $\left|J^{(1)}\right|=o(m / \log m), j=O(m / \log m)$ when $j \in J^{(1)}$, and $m \rightarrow \infty$ as $n \rightarrow \infty$. Then, as $n \rightarrow \infty$,

$$
P_{L}(S, n) \sim\left(\frac{m}{n}\right)^{a-1} \mathscr{S}_{L}(J) \rho^{n-m} \exp \left(-C_{L}(\rho ; J)\right) .
$$


Proof: We know

$$
P_{L}(S, n)=\frac{\left[z^{n}\right] L(z ; S)}{\left[z^{n}\right] L(z)} .
$$

Define restricted patterns $S^{(1)}$ and $S^{(2)}$ such that $S^{(1)}(j)=S(j)$ for $j \in J^{(1)}$ and $S^{(2)}(j)=S(j)$ for $j \in J^{(2)}$. Let $n^{\star}=n-\sum_{j \in J^{(2)}} j S^{(2)}(j)$. Applying Theorem 1 , we obtain

$$
\begin{aligned}
P_{L}(S, n) & =\frac{\left[z^{n}\right] \exp \left(C_{L}(z)-C_{L}(z ; J)\right) \mathscr{S}_{L}(J) z^{n-m}}{\left[z^{n}\right] L(z)} \\
& =\frac{\mathscr{S}_{L}\left(J^{(1)} \cup J^{(2)}\right)\left[z^{m}\right] \exp \left(C_{L}(z)-C_{L}\left(z ; J^{(1)}\right)-C_{L}\left(z ; J^{(2)}\right)\right)}{\left[z^{n^{\star}}\right] L(z)} \frac{\left[z^{n^{\star}}\right] L(z)}{\left[z^{n}\right] L(z)}
\end{aligned}
$$

Since $m<\min \left\{j: j \in J^{(2)}\right\}$, we can remove the term $C_{L}\left(z ; J^{(2)}\right)$ without changing the result, namely,

$$
\left[z^{m}\right] \exp \left(C_{L}(z)-C_{L}\left(z ; J^{(1)}\right)-C_{L}\left(z ; J^{(2)}\right)\right)=\left[z^{m}\right] \exp \left(C_{L}(z)-C_{L}\left(z ; J^{(1)}\right)\right) .
$$

Applying (2), we have

$$
\frac{\left[z^{n^{\star}}\right] L(z)}{\left[z^{n}\right] L(z)} \sim \rho^{n-n^{\star}}\left(\frac{n^{\star}}{n}\right)^{a-1}
$$

Therefore, we obtain

$$
P_{L}(S, n) \sim \mathscr{S}_{L}\left(J^{(2)}\right) \rho^{n-n^{\star}}\left(\frac{n^{\star}}{n}\right)^{a-1} P_{L}\left(S^{(1)}, n^{\star}\right)
$$

For the term $P_{L}\left(S^{(1)}, n^{\star}\right)$, we apply Theorem 1 of (2) to obtain

$$
P_{L}\left(S^{(1)}, n^{\star}\right) \sim\left(\frac{m}{n^{\star}}\right)^{a-1} \mathscr{S}_{L}\left(J^{(1)}\right) \rho^{n^{\star}-m} \exp \left(-C_{L}\left(\rho ; J^{(1)}\right)\right) .
$$

Hence,

$$
\begin{aligned}
P_{L}(S, n) & \sim \mathscr{S}_{L}\left(J^{(2)}\right) \rho^{n-n^{\star}}\left(\frac{n^{\star}}{n}\right)^{a-1}\left(\frac{m}{n^{\star}}\right)^{a-1} \mathscr{S}_{L}\left(J^{(1)}\right) \rho^{n^{\star}-m} \exp \left(-C_{L}\left(\rho ; J^{(1)}\right)\right) \\
& =\mathscr{S}_{L}(J) \rho^{n-m}\left(\frac{m}{n}\right)^{a-1} \exp \left(-C_{L}\left(\rho ; J^{(1)}\right)\right) .
\end{aligned}
$$

This completes the proof of Theorem 3 .

Theorem 4 Let $P_{U}(S, n)$ be the probability that a random decomposable unlabeled structure with size $n$ has a restricted pattern $S$. Suppose $0<\rho<1$. Let $J^{(1)}=\{j \in J: j \leq m\}$ and $J^{(2)}=\{j \in J: j>m\}$. Suppose $\left|J^{(1)}\right|=o(m / \log m), j=O(m / \log m)$ when $j \in J^{(1)}$, and $m \rightarrow \infty$ as $n \rightarrow \infty$. Then, as $n \rightarrow \infty$,

$$
P_{U}(S, n) \sim\left(\frac{m}{n}\right)^{a-1} \mathscr{S}_{U}(J) \rho^{n-m} \prod_{j \in J^{(1)}}\left(1-\rho^{j}\right)^{C_{j}} .
$$


Proof: The proof is similar to the one of Theorem 3 .

\section{The $r$ th Smallest Component with a Given Restricted Pattern}

In this section we present our main results about the size of the $r$ th smallest component in a random decomposable structure with a given restricted pattern. Our results extend those of Panario and Richmond (14).

Let $X_{n}^{[r]}(S)$ denote the size of the $r$ th smallest component of a random decomposable combinatorial structure of size $n$ having a restricted pattern $S$. We derive an asymptotic expression for $P\left(X_{n}^{[r]}(S)>k\right)$. We observe that $P\left(X_{n}^{[r]}(S)>k\right)=0$ if $d(k) \geq r$ where $d(k)$ is defined in Section 2 .

\subsection{Labeled Case}

In the following, we adopt the convention $0^{0}=1$ and use the notations $J^{(1)}$ and $J^{(2)}$ which are defined in Theorems 3 and 4 .

Theorem 5 Let $S$ be a restricted pattern with $\left|J^{(1)}\right|=o(m / \log m)$. Assume $j=O(m / \log m)$ when $j \in J^{(1)}, r=O(\log m), k=o(m / \log m)$ and $m \rightarrow \infty$. If $k$ is bounded, we have

$$
P\left(X_{n}^{[r]}(S)>k\right) \sim P_{L}(S, n) \exp \left(-C_{L}\left(\rho ; N_{k} \backslash J\right)\right) \sum_{j=0}^{r-1-d(k)} \frac{\left(C_{L}\left(\rho ; N_{k} \backslash J\right)\right)^{j}}{j !} .
$$

If $k \rightarrow \infty$ (as $n \rightarrow \infty)$, letting $G(k)=a \log (k)+a \gamma+K-C\left(\rho ; J \cap N_{k}\right)$, we have

$$
P\left(X_{n}^{[r]}(S)>k\right) \sim P_{L}(S, n) \frac{\exp \left(C_{L}\left(\rho ; J \cap N_{k}\right)\right)}{k^{a} \exp (a \gamma+K)} \sum_{j=0}^{r-1-d(k)} \frac{(G(k))^{j}}{j !} .
$$

\section{Proof:}

To keep track of the size of the $r$ th smallest component, it is convenient to consider augmented patterns $S^{\star}: J^{\star} \mapsto N$ where $J^{\star}=J \cup N_{k}, S^{\star}(j)=S(j)$ for $j \in J$, and, for $j \in N_{k} \backslash J, S^{\star}(j)$ is specified below. We note that each structure with the restricted pattern $S$ such that its $r$ th smallest component has size greater than $k$ corresponds to a structure with an augmented pattern $S^{\star}$ such that $\sum_{i \in N_{k} \backslash J} S^{\star}(i) \leq r-1-d(k)$. Hence we have

$$
P\left(X_{n}^{[r]}(S)>k\right)=\sum_{\sum_{i \in N_{k} \backslash J} S^{\star}(i)=0}^{r-1-d(k)} P_{L}\left(S^{\star}\right) .
$$

Let $m^{\star}=n-\sum_{j \in J \cup N_{k}} j S^{\star}(j)$. Since $k=o(m / \log m)$ and $\sum_{j \in N_{k} \backslash J} S^{\star}(j) \leq r-1-d(k)=$ $O(\log m)$, we have $\frac{m^{\star}}{n} \sim \frac{m}{n}$. Applying Theorem 3 and noticing that $\sum_{j \in J \cap N_{k}} j S(j)$ is given by 
the pattern $S$, we get

$$
\begin{aligned}
& P\left(X_{n}^{[r]}(S)>k\right) \\
\sim & \sum_{\sum_{j \in N_{k} \backslash J} S^{\star}(j)=0}^{r-1-d(k)}\left(\frac{m^{\star}}{n}\right)^{a-1} \prod_{i \in J \cup N_{k}} \frac{C_{i}^{S^{\star}(i)}}{(i !)^{S^{\star}(i)} S^{\star}(i) !} \rho^{n-m^{\star}} \exp \left(-C_{L}\left(\rho ; J^{(1)} \cup N_{k}\right)\right) \\
\sim & \left(\frac{m}{n}\right)^{a-1} \mathscr{S}_{L}(J) \rho^{n-m} \exp \left(-C_{L}\left(\rho ; J^{(1)}\right)-C_{L}\left(\rho ; N_{k} \backslash J\right)\right) \\
& \quad \sum^{r-1-d(k)}\left(\prod_{j \in N_{k} \backslash J} \frac{C_{i}^{S^{\star}(i)} \rho^{i S^{\star}(i)}}{(i !)^{S^{\star}(i)} S^{\star}(i) !}\right) \\
\sim & P_{L}(S, n) \exp \left(-C_{L}\left(\rho ; N_{k} \backslash J\right)\right) \sum_{\sum_{j \in N_{k} \backslash J} S^{\star}(j)=0}^{r-1-d(k)}\left(\prod_{i \in N_{k} \backslash J} \frac{C_{i}^{S^{\star}(i)} \rho^{i S^{\star}(i)}}{(i !)^{S^{\star}(i)} S^{\star}(i) !}\right) .
\end{aligned}
$$

It follows from (3) and the multinomial formula

$$
\begin{aligned}
\left(Y_{1}+Y_{2}+\cdots+Y_{k}\right)^{j} & =\sum_{a_{1}+a_{2}+\cdots+a_{k}=j}\left(\begin{array}{c}
j \\
a_{1}, a_{2}, \ldots, a_{k}
\end{array}\right) Y_{1}^{a_{1}} Y_{2}^{a_{2}} \ldots Y_{k}^{a_{k}} \\
& =j ! \sum_{a_{1}+a_{2}+\cdots+a_{k}=j} \prod_{i=1}^{k} \frac{Y_{i}^{a_{i}}}{a_{i} !}
\end{aligned}
$$

that

$$
P\left(X_{n}^{[r]}(S)>k\right) \sim P_{L}(S, n) \exp \left(-C_{L}\left(\rho ; N_{k} \backslash J\right)\right) \sum_{j=0}^{r-1-d(k)} \frac{\left(C_{L}\left(\rho ; N_{k} \backslash J\right)\right)^{j}}{j !} .
$$

If $k$ is bounded, we are done. If $k \rightarrow \infty$, we have, using the transfer theorem again,

$$
\begin{aligned}
C_{L}\left(\rho ; N_{k}\right) & =\sum_{i=1}^{k} C_{i} \rho^{i} / i !=\left[z^{k}\right] \frac{1}{1-z} C(\rho z) \\
& =\left[z^{k}\right] \frac{a}{1-z} \log \frac{1}{1-z}+K+O\left(k^{-\alpha}\right) \\
& =a \log (k)+a \gamma+K+O\left(k^{-\alpha}\right),
\end{aligned}
$$

where $\gamma$ is the Euler-Mascheroni constant.

Let $G(k)=a \log (k)+a \gamma+K-C_{L}\left(\rho ; J \cap N_{k}\right)$. Then

$$
P\left(X_{n}^{[r]}(S)>k\right) \sim P_{L}(S, n) \frac{\exp \left(C_{L}\left(\rho ; J \cap N_{k}\right)\right)}{k^{a} \exp (a \gamma+K)} \sum_{j=0}^{r-1-d(k)} \frac{(G(k))^{j}}{j !} .
$$


When there is no restricted pattern, let $X_{n}^{[r]}$ denote the size of the $r$ th smallest component of a labeled decomposable structure of size $n$. From Theorem 5 we get the following result of Panario and Richmond (14).

Corollary 1 Let $r=O(\log n), k=o(n / \log n)$ and $n \rightarrow \infty$. If $k$ is bounded, we have

$$
P\left(X_{n}^{[r]}>k\right) \sim \exp \left(-C_{L}\left(\rho ; N_{k}\right)\right) \sum_{j=0}^{r-1} \frac{C_{L}\left(\rho ; N_{k}\right)^{j}}{j !} .
$$

If $k \rightarrow \infty$, we have

$$
\left.P\left(X_{n}^{[r]}>k\right) \sim k^{-a} \exp (-a \gamma-K)\right)\left(\sum_{j=0}^{r-1} \frac{(a \log k)^{j}}{j !}\right) .
$$

The expression in Theorem 5 can be simplified when $r-d(k)$ and $\left|J \cap N_{k}\right|$ are small compared with $k$.

Corollary 2 Using the same notations and conditions as those in Theorem 5 , if we further limit $\log \left|J \cap N_{k}\right|=o(\log k)$ and $r-d(k)=o(\log k)$ with $k \rightarrow \infty$, we have, as $m \rightarrow \infty$,

$$
P\left(X_{n}^{[r]}(S)>k\right) \sim P_{L}(S, n) \frac{\exp \left(C_{L}\left(\rho ; J \cap N_{k}\right)\right)}{k^{a} \exp (a \gamma+K)} \frac{(a \log (k))^{r-1-d(k)}}{(r-1-d(k)) !} .
$$

Proof:

Since $\log \left|J \cap N_{k}\right|=o(\log k)$ and $C_{L}\left(\rho ; J \cap N_{k}\right)=O\left(C_{L}\left(\rho ; N_{\left|J \cap N_{k}\right|}\right)\right)$, applying Equation (4), we get

$$
C_{L}\left(\rho ; J \cap N_{k}\right)=o(\log k)
$$

Hence, $a \log (k)+a \gamma+K-C_{L}\left(\rho ; J \cap N_{k}\right)=a \log k(1+o(1))$.

From $r-d(k)=o(\log k)$, we have

$$
\sum_{j=0}^{r-1-d(k)} \frac{\left(C_{L}\left(\rho ; N_{k} \backslash J\right)\right)^{j}}{j !} \sim \frac{(a \log k)^{r-1-d(k)}}{(r-1-d(k)) !} .
$$

Now the corollary follows immediately from Theorem 5

\subsection{Unlabeled Case}

In the unlabeled case we assume $0<\rho<1$.

Theorem 6 Let $S$ be a restricted pattern with $\left|J^{(1)}\right|=o(m / \log m)$. Assume $j=O(m / \log m)$ when $j \in J^{(1)}, r=O(\log m)$ and $k=o(m / \log m)$. As $m \rightarrow \infty$, we have, if $k$ is bounded,

$P\left(X_{n}^{[r]}(S)>k\right) \sim P_{U}(S, n) h\left(\rho ; N_{k} \backslash J\right) \sum_{j=0}^{r-1-d(k)}\left[z^{j}\right] \exp \left(z C_{U}\left(\rho ; N_{k} \backslash J\right)+z^{2} \frac{C_{U}\left(\rho^{2} ; N_{k} \backslash J\right)}{2}+\cdots\right)$. 
If $k \rightarrow \infty$, we have

$P\left(X_{n}^{[r]}(S)>k\right) \sim P_{U}(S, n) \frac{e^{-a \gamma-K-r_{0}}}{k^{a} h\left(\rho ; J \cap N_{k}\right)} \sum_{j=0}^{r-1-d(k)}\left[z^{j}\right] \exp \left(z C_{U}\left(\rho ; N_{k} \backslash J\right)+z^{2} \frac{C_{U}\left(\rho^{2} ; N_{k} \backslash J\right)}{2}+\cdots\right)$.

Proof:

We define the same augmented pattern $S^{\star}$ as the one in the proof of Theorem 5 . Let $m^{\star}=$ $n-\sum_{j \in J \cup N_{k}} j S^{\star}(j)$. We still have $\frac{m^{\star}}{n} \sim \frac{m}{n}$ for the same reason as in the labeled case. Applying Theorem 4 , we get

$$
\begin{aligned}
P\left(X_{n}^{[r]}(S)>k\right) & \sim \sum_{\sum_{j \in N_{k} \backslash J} S^{\star}(j)=0}^{r-1-d(k)}\left(\frac{m^{\star}}{n}\right)^{a-1} \prod_{j \in J \cup N_{k}}\left(\begin{array}{c}
C_{j}+S^{\star}(j)-1 \\
S^{\star}(j)
\end{array}\right) \prod_{j \in J^{(1) \cup N_{k}}}\left(1-\rho^{j}\right)^{C_{j}} \rho^{n-m^{\star}} \\
& \sim P_{U}(S, n) h\left(\rho ; N_{k} \backslash J\right) \sum_{\sum_{j \in N_{k} \backslash J} S^{\star}(j)=0}^{r-1-d(k)} \prod_{j \in N_{k} \backslash J}\left(\begin{array}{c}
C_{j}+S^{\star}(j)-1 \\
S^{\star}(j)
\end{array}\right) \rho^{j S^{\star}(j)}
\end{aligned}
$$

The negative binomial theorem states that

$$
\left(1-\rho^{i} z\right)^{-C_{i}}=\sum_{X_{i} \geq 0}\left(\begin{array}{c}
C_{i}+X_{i}-1 \\
X_{i}
\end{array}\right) \rho^{i X_{i}} z^{X_{i}}
$$

Hence, we get

$$
\begin{aligned}
\sum_{\sum_{j \in N_{k} \backslash J} S^{\star}(j)=0}^{r-1-d(k)} \prod_{j \in N_{k} \backslash J}\left(\begin{array}{c}
C_{j}+S^{\star}(j)-1 \\
S^{\star}(j)
\end{array}\right) \rho^{j S^{\star}(j)} & =\sum_{u=0}^{r-1-d(k)}\left[z^{u}\right] \prod_{j \in N_{k} \backslash J}\left(1-\rho^{j} z\right)^{-C_{j}} \\
& =\sum_{u=0}^{r-1-d(k)}\left[z^{u}\right] \exp \left(-\sum_{j \in N_{k} \backslash J} C_{j} \log \left(1-\rho^{j} z\right)\right) \\
& =\sum_{u=0}^{r-1-d(k)}\left[z^{u}\right] \exp \left(\sum_{i \geq 1} \sum_{j \in N_{k} \backslash J} C_{j} \rho^{i j} z^{i} / i\right) \\
& =\sum_{u=0}^{r-1-d(k)}\left[z^{u}\right] \exp \left(z\left(C_{U}\left(\rho ; N_{k}\right)-C_{U}\left(\rho ; J \cap N_{k}\right)\right)\right. \\
& \left.+z^{2} \frac{C_{U}\left(\rho^{2} ; N_{k}\right)-C_{U}\left(\rho^{2} ; J \cap N_{k}\right)}{2}+\cdots\right) . \quad(6)
\end{aligned}
$$

When $k \rightarrow \infty$, by (4), we have

$$
C_{U}\left(\rho ; N_{k}\right)=a \log (k)+a \gamma+K+O\left(k^{-\alpha}\right) .
$$

Since $\rho$ is the radius of convergence of $C_{U}(z)$, and in the unlabeled case $0<\rho<1, C_{U}\left(\rho^{t}\right)$ is bounded for an integer $t \geq 2$. We have that $C_{U}\left(\rho^{t} ; N_{k}\right) \sim C_{U}\left(\rho^{t}\right)$ as $k \rightarrow \infty$. 
Similarly to the derivation of (4) and the above discussion, we get

$$
\begin{aligned}
h\left(\rho ; N_{k}\right) & =\exp \left(\sum_{i=1}^{k} C_{i} \log \left(1-\rho^{i}\right)\right) \sim \exp \left(-\sum_{i=1}^{k} C_{i} \rho^{i}\right) e^{-r_{0}} \\
& \sim k^{-a} \exp \left(-a \gamma-K-r_{0}\right),
\end{aligned}
$$

where $r_{0}=\frac{C_{U}\left(\rho^{2}\right)}{2}+\frac{C_{U}\left(\rho^{3}\right)}{3}+\cdots$. Finally, in Equation 5 , we do the following substitution

$$
h\left(\rho ; N_{k} \backslash J\right)=\frac{h\left(\rho ; N_{k}\right)}{h\left(\rho ; J \cap N_{k}\right)},
$$

and the proof is complete.

As in the labeled case, we provide the following two corollaries. The first one is for the special case $J=\emptyset$.

Corollary 3 Let $r=o(\log n)$ and $k=o(n / \log n)$. Using the same notations as in Theorem 6 , we have, if $k$ is bounded,

$$
P\left(X_{n}^{[r]}>k\right) \sim h\left(\rho ; N_{k}\right) \sum_{j=0}^{r-1-d(k)}\left[z^{j}\right] \exp \left(z C_{U}\left(\rho ; N_{k}\right)+z^{2} \frac{C_{U}\left(\rho^{2} ; N_{k}\right)}{2}+\cdots\right) .
$$

If $k \rightarrow \infty$, we have

$$
P\left(X_{n}^{[r]}>k\right) \sim \frac{e^{-a \gamma-K-r_{0}}}{k^{a}} \sum_{j=0}^{r-1-d(k)}\left[z^{j}\right] \exp \left(a z \log k+z^{2} \frac{C_{U}\left(\rho^{2}\right)}{2}+\cdots\right) .
$$

Corollary 4 Assume the same notations and conditions as in Theorem 6 . Assume further $\log \left|J \cap N_{k}\right|=o(\log k)$ and $r-d(k)=o(\log k)$ as $k \rightarrow \infty$. We then have

$$
P\left(X_{n}^{[r]}(S)>k\right) \sim P_{U}(S, n) \frac{(a \log k)^{r-1-d(k)}}{(r-1-d(k)) ! k^{a} e^{a \gamma+K+r_{0}} h\left(\rho ; J \cap N_{k}\right)} .
$$

Proof:

Since $\log \left|J \cap N_{k}\right|=o(\log k)$ and $C_{U}\left(\rho ; J \cap N_{k}\right)=O\left(C_{U}\left(\rho ; N_{\left|J \cap N_{k}\right|}\right)\right)$, applying (4), we have

$$
C_{U}\left(\rho ; J \cap N_{k}\right)=o(\log k)
$$

Hence,

$$
C_{U}\left(\rho ; N_{k} \backslash J\right)=a \log k(1+o(1)) .
$$

From (6), noticing that $r-d(k)=o(\log k)$, we have

$$
\begin{aligned}
\sum_{j=0}^{r-1-d(k)}\left[z^{j}\right] \exp \left(z\left(C_{U}\left(\rho ; N_{k} \backslash J\right)\right)+z^{2} \frac{C_{U}\left(\rho^{2} ; N_{k} \backslash J\right)}{2}+\cdots\right) & \sim \sum_{j=0}^{r-1-d(k)}\left[z^{j}\right] \exp (a z \log k) \\
& \sim \frac{(a \log k)^{r-1-d(k)}}{(r-1-d(k)) !} .
\end{aligned}
$$


Therefore, the corollary follows immediately from Theorem 6 .

\section{First Moment}

In this section, given a restricted pattern $S: J \mapsto N$, we consider the asymptotic behavior of the average size of the $r$ th smallest component. We only discuss the pattern when $J^{(2)}=\emptyset$ where $J^{(2)}$, defined in Section 2 , is the set of the sizes of large components in the pattern $S$.

Let $E\left(X_{n}^{[r]}(S)\right)$ stand for the first moment of $X_{n}^{[r]}(S)$. To estimate the first moment, we use the well-known expression

$$
E\left(X_{n}^{[r]}(S)\right)=\sum_{k \geq 0} P\left(X_{n}^{[r]}(S)>k\right)
$$

Let $i^{[j]}$ denote the size of the $j$ th component in the given restricted pattern. If $r \leq \sum_{j \in J} S(j)$, it is clear that $P\left(X_{n}^{[r]}(S)>k\right)=0$ when $\sum_{j \in J \cap N_{k}} S(j) \geq r$. Hence we have

$$
E\left(X_{n}^{[r]}(S)\right)=\sum_{k=0}^{i^{[r]}-1} P\left(X_{n}^{[r]}>k\right) .
$$

If $r>\sum_{j \in J} S(j)$, we need to estimate $P\left(X_{n}^{[r]}(S)>k\right)$ when $k$ exceeds the range given in Theorem 5 .

Let $\lfloor x\rfloor$ be the biggest integer which is smaller than or equal to $x$. We shall estimate $E\left(X_{n}^{[r]}(S)\right)$ under either of the following two conditions

(C1) $a>1, r=o\left(\frac{\log n}{\log \log n}\right)$ and $\log |J|=o(\log n)$,

(C2) $a=1, r=O(1)$ and $|J|=O(\log n)$.

To prove later theorems, we introduce the following lemma.

Lemma 1 Let $a, b$, and $c$ be positive integers. Suppose $a \rightarrow \infty, \frac{\log b}{\log a} \rightarrow \infty$, and $c=O(1)$. Then,

$$
\sum_{k=a}^{b} \frac{(\log k)^{c}}{k} \sim \frac{1}{c+1}(\log b)^{c+1} .
$$

Proof:

Let us consider the continuous real function $f(x)=\frac{1}{x}(\log x)^{c}$ where $x \in[a, b]$. We have

$$
f^{\prime}(x)=\frac{1}{x^{2}}(c-\log x)(\log x)^{c-1}<0,
$$

since $c$ is a constant and $a \rightarrow \infty$. Hence, $f(x)$ is monotonic decreasing and we have

$$
\int_{a}^{b} f(k) d k<\sum_{k=a}^{b} \frac{(\log k)^{c}}{k}<\int_{a}^{b} f(k-1) d k,
$$


and the proof is done since

$$
\int_{a}^{b} f(k) d k \sim \int_{a}^{b} f(k-1) d k \sim \frac{1}{c+1}(\log b)^{c+1} .
$$

For a random decomposable combinatorial structure with a given restricted pattern, the first moment of the $r$ th smallest component is given by the following theorem.

Theorem 7 Let $M=\max \{j: j \in J\}$. Assume $r>\sum_{j \in J} S(j)$ and $M=o(n / \log n)$. Define $w=\max \left\{\left\lfloor\frac{n}{\log n \log \log n}\right\rfloor, M\right\}$. Under either $(C 1)$ or $(C 2)$ conditions, and as $m \rightarrow \infty$, for both labeled and unlabeled cases, we have

$$
E\left(X_{n}^{[r]}(S)\right) \sim \sum_{k=0}^{w} P\left(X_{n}^{[r]}(S)>k\right)
$$

\section{Proof:}

We only prove this theorem for the labeled case since the proof of the unlabeled case is very similar.

We start with the condition (C1), that is, $a>1, r=o(\log n / \log \log n)$ and $\log |J|=o(\log n)$. From the definition, we have $P\left(X_{n}^{[r]}(S)>k\right)>0$, for any positive $k$. According to Theorem 5 we have

$$
\sum_{k=0}^{w} P\left(X_{n}^{[r]}(S)>k\right)>P\left(X_{n}^{[r]}(S)>0\right)=P_{L}(S, n) .
$$

Let $\hat{d}=\sum_{j \in J} S(j)$. From Corollary 2 , we have

$$
\begin{aligned}
\sum_{k=w+1}^{n} P\left(X_{n}^{[r]}(S)>k\right) & \leq n P\left(X_{n}^{[r]}(S)>w\right) \\
& \sim n P_{L}(S, n) O\left(\frac{\exp \left(C_{L}\left(\rho ; J \cap N_{w}\right)\right)(a \log w)^{r-1-\hat{d}}}{(r-1-\hat{d}) ! w^{a}}\right) \\
& \leq n P_{L}(S, n) O\left(\frac{\exp \left(C_{L}\left(\rho ; J \cap N_{w}\right)\right) a^{r-1-\hat{d}}(\log \log n)^{a}(\log n)^{r+a-1-\hat{d}}}{(r-1-\hat{d}) ! n^{a}}\right) .
\end{aligned}
$$

In the last line, we use $w \geq\left\lfloor\frac{n}{\log n \log \log n}\right\rfloor$. In this case, the constant $a$ is bigger than 1 . According to condition $(C 1)$, we have

$$
\exp \left(C_{L}\left(\rho ; J \cap N_{w}\right)\right)=O\left(\exp \left(C_{L}\left(\rho ; N_{|J|}\right)\right)\right)=O(\exp (o(\log n)))=O\left(n^{o(1)}\right),
$$

and

$$
(\log n)^{r+a-1-\hat{d}}=(\log n)^{o\left(\frac{\log n}{\log \log n}\right)}=n^{o(1)} .
$$


Hence,

$$
\sum_{k=w+1}^{n} P\left(X_{n}^{[r]}(S)>k\right)=P_{L}(S, n) o(1)
$$

which is negligible compared with $\sum_{k=0}^{w} P\left(X_{n}^{[r]}(S)>k\right)$.

We consider now condition $(C 2)$, that is, $a=1, r=O(1)$ and $|J|=\log n$. We divide this proof into three parts. In the first part, we prove that

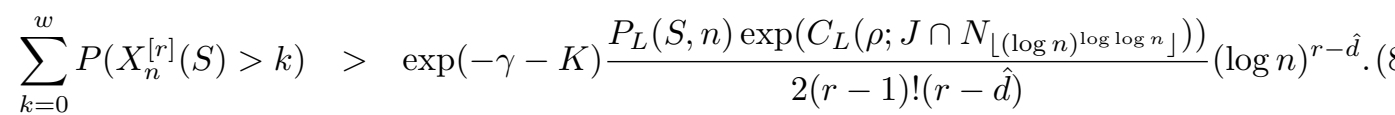

In the second part, we prove

$$
\sum_{k=w}^{\lfloor n / \log n\rfloor} P\left(X_{n}^{[r]}(S)>k\right)=o\left(P_{L}(S, n) \exp \left(C_{L}\left(\rho ; J \cap N_{\left\lfloor(\log n)^{\log \log n\rfloor}\right.}\right)\right)(\log n)^{r-\hat{d}}\right) .
$$

In the third part, we explain that $\sum_{k=n / \log n}^{n} P\left(X_{n}^{[r]}(S)>k\right)$ is negligible compared with $P_{L}(S, n)$ $\exp \left(C_{L}\left(\rho ; J \cap N_{\left\lfloor(\log n)^{\log \log n}\right\rfloor}\right)\right)(\log n)^{r-\hat{d}}$.

Before we start the proof, let us first estimate $C_{L}\left(\rho ; J \cap N_{k}\right)$ when $k>(\log n)^{\log \log n}$. Under the condition $(C 2),|J|=O(\log n)$. Then we have

$$
\begin{aligned}
C_{L}\left(\rho ; J \cap N_{k}\right) & =C_{L}\left(\rho ; J \cap N_{(\log n)^{\log \log n}}\right)+C_{L}\left(\rho ; J \cap\left(N_{k} \backslash N_{\left.(\log n)^{\log \log n}\right)}\right)\right. \\
& \leq C_{L}\left(\rho ; J \cap N_{(\log n)^{\log \log n}}\right)+C_{L}\left(\rho ;\left(N_{(\log n)^{\log \log n}+|J|} \backslash N_{\left.(\log n)^{\log \log n}\right)}\right)\right. \\
& =C_{L}\left(\rho ; J \cap N_{(\log n)^{\log \log n}}\right)+o(1) .
\end{aligned}
$$

Part I: proof of (8).

Applying Lemma 1 and Corollary 2, we get

$$
\begin{aligned}
& \sum_{k=0}^{w} P\left(X_{n}^{[r]}(S)>k\right)>\sum_{k=\left\lfloor(\log n)^{\log \log n}\right.}^{w} P\left(X_{n}^{[r]}(S)>k\right) \\
& >\frac{\exp (-\gamma-K)}{2(r-1) !} \exp \left(C _ { L } \left(\rho ; J \cap N_{\left.\left\lfloor(\log n)^{\log \log n\rfloor}\right)\right) P_{L}(S, n)} \sum_{k=\left\lfloor(\log n)^{\log \log n}\right.}^{w} \frac{(\log k)^{r-1-\hat{d}}}{k}\right.\right. \\
& \sim \exp (-\gamma-K) \frac{P_{L}(S, n) \exp \left(C _ { L } \left(\rho ; J \cap N_{\left.\left\lfloor(\log n)^{\log \log n\rfloor}\right)\right)}\right.\right.}{2(r-1) !(r-\hat{d})}(\log n)^{r-\hat{d}} .
\end{aligned}
$$

Part II: proof of 9 . 
Decomposable Structures with a Restricted Pattern

Applying that $r=O(1)$ and Corollary 2, we obtain

$$
\begin{aligned}
\sum_{k=w+1}^{\left\lfloor\frac{n}{\log n}\right\rfloor} P\left(X_{n}^{[r]}(S)>k\right) & <\frac{n}{\log n} P\left(X_{n}^{[r]}(S)>w\right) \\
& \leq \frac{n}{\log n} P_{L}(S, n) O\left(\frac{\exp \left(C_{L}\left(\rho ; J \cap N_{w}\right)\right) \log \log n(\log n)^{r-\hat{d}}}{n}\right) .
\end{aligned}
$$

Now applying (10), we get

$$
C\left(\rho ; J \cap\left(N_{w} \backslash N_{\left\lfloor(\log n)^{\log \log n}\right\rfloor}\right)\right)=o(1) .
$$

Putting this back to (12), we have

$$
\sum_{k=w+1}^{\left\lfloor\frac{n}{\log n}\right\rfloor} P\left(X_{n}^{[r]}(S)>k\right)=P_{L}(S, n) o\left(\exp \left(C_{L}\left(\rho ; J \cap N_{\left\lfloor(\log n)^{\log \log n}\right\rfloor}\right)\right)(\log n)^{r-\hat{d}}\right) .
$$

Part III: we prove that $\sum_{k=n / \log n}^{n} P\left(X_{n}^{[r]}(S)>k\right)$ is negligible.

Let $\varepsilon=\frac{1}{\log \log \log n}$ and $w^{\prime}=\frac{\sqrt{\varepsilon} n}{\log n}$. We have

$$
\varepsilon n \frac{\left(\log w^{\prime}\right)^{r-\hat{d}-1}}{w^{\prime}}=o\left((\log n)^{r-\hat{d}}\right) .
$$

Similar to (13), we get

$$
\begin{aligned}
\sum_{k=w^{\prime}}^{\lfloor\varepsilon n\rfloor} P\left(X_{n}^{[r]}(S)>k\right) & =O\left(\varepsilon n P_{L}(S, n) \exp \left(C_{L}\left(\rho ; J \cap N_{w^{\prime}}\right)\right) \frac{\left(\log w^{\prime}\right)^{r-\hat{d}-1}}{w^{\prime}}\right) \\
& \left.=P_{L}(S, n) o\left(C_{L}\left(\rho ; N_{\left\lfloor(\log n)^{\log \log n\rfloor}\right.}\right)\right)(\log n)^{r-\hat{d}}\right) .
\end{aligned}
$$

In order to estimate $P\left(X_{n}^{[r]}(S)>k\right)$ when $k=\lfloor\varepsilon n\rfloor$, we have to go back to the proof of Theorem 5. Let $\mathbb{S}$ be the set of augmented patterns of $S$ as defined in the proof of Theorem 3 . For any $S^{\star} \in \mathbb{S}$, the corresponding $J^{\star}$ satisfies $J^{\star}=N_{\lfloor\varepsilon n\rfloor}$ and $0 \leq \sum_{j \in J^{\star} \backslash J} S^{\star}(j) \leq r-\hat{d}-1$. We need to estimate $P_{L}\left(S^{\star}, n\right)$ for every $S^{\star} \in \mathbb{S}$. We notice that $J \subset N_{\lfloor\varepsilon n\rfloor}$. We base our analysis on $(C 2)$. Given $a=1$, we get the expression of $P_{L}\left(S^{\star}, n\right)$

$$
\begin{aligned}
P_{L}\left(S^{\star}, n\right) & \sim \mathscr{S}_{L} \rho^{n-m^{\star}} e^{-K} \prod_{j \in N_{\lfloor\varepsilon n\rfloor J}} \frac{C_{j}^{S^{\star}(j)}}{(j !)^{S^{\star}(j)} S^{\star}(j) !}\left[z^{m^{\star}}\right] \exp \left(\sum_{j=\lfloor\varepsilon n\rfloor+1}^{n} \frac{z^{j}}{j}\right) \\
& \sim \mathscr{S}_{L} \rho^{n-m} e^{-K} \prod_{j \in N_{\lfloor\varepsilon n\rfloor J}} \frac{\left(C_{j} \rho^{j}\right)^{S^{\star}(j)}}{(j !)^{S^{\star}(j)} S^{\star}(j) !}\left[z^{m^{\star}}\right] \prod_{j=\lfloor\varepsilon n\rfloor+1}^{n}\left(\sum_{i \geq 0} \frac{z^{i j}}{i ! j}\right) .
\end{aligned}
$$


Recall that $m=n-\sum_{j \in J} j S(j)$ for a given pattern $S$. For any $S^{\star} \in \mathbb{S}$, we have $n / m^{\star} \rightarrow 1$ since $r>\hat{d}$ and $r=O(1)$. Let $S^{\prime}: J^{\prime} \mapsto N$ be an augmented pattern belonging to $\mathbb{S}$ and the corresponding $m^{\prime}=n-\sum_{j \in J^{\prime}} j S^{\prime}(j)$ such that, for any $S^{\star} \in \mathbb{S}$, we have

$$
\left[z^{m^{\star}}\right] \prod_{j=\lfloor\varepsilon n\rfloor+1}^{n}\left(\sum_{i \geq 0} \frac{z^{i j}}{i ! j^{i}}\right) \leq\left[z^{m^{\prime}}\right] \prod_{j=\lfloor\varepsilon n\rfloor+1}^{n}\left(\sum_{i \geq 0} \frac{z^{i j}}{i ! j^{i}}\right) .
$$

Following a similar reasoning as the one we used in the proof of Theorem 3 to derive $P\left(X_{n}^{[r]}(S)>\right.$ $k)$, we obtain

$$
\begin{aligned}
& P\left(X_{n}^{[r]}(S)>\lfloor\varepsilon n\rfloor\right)=\sum_{\sum_{i \in N\lfloor\varepsilon n\rfloor J} S^{\star}(i)=0}^{r-1-\hat{d}} P_{L}\left(S^{\star}, n\right) \\
& \leq \mathscr{S}_{L} \rho^{n-m} e^{-K}\left[z^{m^{\prime}}\right] \prod_{j=\lfloor\varepsilon n\rfloor+1}^{m^{\prime}}\left(\sum_{i \geq 0} \frac{z^{i j}}{i ! j^{i}}\right) \sum_{\sum_{i \in N\lfloor\varepsilon n\rfloor J} S^{\star}(i)=0}^{r-1-\hat{d}} \prod_{j \in N_{\lfloor\varepsilon n\rfloor \backslash}} \frac{\left(C_{j} \rho^{j}\right)^{S^{\star}(j)}}{(j !)^{S^{\star}(j)} S^{\star}(j) !} .
\end{aligned}
$$

Again, using the same method as in the proof of Theorem 5. we have

$$
\sum_{\sum_{i \in N_{\lfloor\varepsilon n\rfloor} \backslash J}^{r-1-\hat{d}} S^{\star}(i)=0} \prod_{j \in N_{\lfloor\varepsilon n\rfloor} \backslash J} \frac{\left(C_{j} \rho^{j}\right)^{S^{\star}(j)}}{(j !)^{S^{\star}(j)} S^{\star}(j) !}=\sum_{i=0}^{r-1-\hat{d}} \frac{\left(C\left(\rho ; N_{\lfloor\varepsilon n\rfloor} \backslash J\right)\right)^{i}}{i !}=O\left((\log n)^{r-1-\hat{d}}\right) .
$$

Hence, we obtain

$$
\begin{aligned}
P\left(X_{n}^{[r]}(S)>\lfloor\varepsilon n\rfloor\right) & =O\left(\mathscr{S}_{L} \rho^{n-m}(\log n)^{r-1-\hat{d}}\left[z^{m^{\prime}}\right] \prod_{j=\lfloor\varepsilon n\rfloor+1}^{m^{\prime}}\left(\sum_{i \geq 0} \frac{z^{i j}}{i ! j^{i}}\right)\right) \\
& =O\left(\mathscr{S}_{L} \rho^{n-m}(\log n)^{r-1-\hat{d}} \sum_{\substack{\sum_{j=\lfloor\varepsilon n\rfloor+1}^{m^{\prime}} i_{j j} j=m^{\prime} \\
j=\lfloor\varepsilon n\rfloor+1}}^{m^{\prime}} \frac{1}{i_{j} ! j^{i_{j}}}\right) \\
& =O\left(\mathscr{S}_{L} \rho^{n-m}(\log n)^{r-1-\hat{d}} \sum_{h=1}^{\lfloor 1 / \varepsilon\rfloor} \sum_{\substack{\sum_{j=\lfloor\varepsilon n\rfloor+1}^{m^{\prime}} i_{j}=h \\
\sum_{j=\lfloor\varepsilon n\rfloor+1}^{m^{\prime}} i_{j} j=m^{\prime}}} \prod_{j=\lfloor\varepsilon n\rfloor+1}^{m^{\prime}} \frac{1}{i_{j} ! j^{i_{j}}}\right)
\end{aligned}
$$

Since $\varepsilon=1 / \log \log \log n$, we have

$$
\left(\frac{1}{\varepsilon}\right)^{\left\lfloor\frac{1}{\varepsilon}\right\rfloor+1}=o(\log n)
$$


If $\sum_{j=\lfloor\varepsilon n\rfloor+1}^{m^{\prime}} i_{j}=h$,

$$
\prod_{j=\lfloor\varepsilon n\rfloor+1}^{m^{\prime}} \frac{1}{i_{j} ! j^{i_{j}}}=O\left(\frac{1}{(\varepsilon n)^{h}}\right)
$$

For integer $0<k \leq n$, let $p_{n}(k)$ denote the number of solutions, in positive integers, to $x_{1}+x_{2}+$ $\cdots+x_{k}=n$. It is well-known that $p_{n}(k)=\left(\begin{array}{c}n-1 \\ k-1\end{array}\right)=O\left(n^{k-1}\right)$.

Applying (17) in (15), since $p_{m^{\prime}}(h)$ is the upper bound of

$$
\sum_{\substack{\sum_{j=\lfloor\varepsilon n\rfloor+1}^{m^{\prime}} \\ \sum_{j=\lfloor\varepsilon n\rfloor+1}^{m^{\prime}} i_{j} j=m^{\prime}}} 1,
$$

we obtain

$$
\sum_{\substack{\sum_{j=\lfloor\varepsilon n\rfloor+1}^{m^{\prime}} \\ \sum_{j=\lfloor\varepsilon n\rfloor+1}^{m^{\prime}} i_{j} j=m^{\prime}}} \prod_{j=\lfloor\varepsilon n\rfloor+1}^{m^{\prime}} \frac{1}{i_{j} ! j^{i_{j}}} \leq O\left(\frac{1}{(\varepsilon n)^{h}}\right) p_{m^{\prime}}(h)=O\left(\frac{1}{(\varepsilon n)^{h}} n^{h-1}\right) .
$$

In the last line, we use $n^{h-1}$ instead of $\left(m^{\prime}\right)^{h-1}$ since $m^{\prime} \leq n$. Hence, applying (16), we have

$$
P\left(X_{n}^{[r]}(S)>\lfloor\varepsilon n\rfloor\right)=O\left(\mathscr{S}_{L} \rho^{n-m}(\log n)^{r-1-\hat{d}} \sum_{h=1}^{\left\lfloor\frac{1}{\varepsilon}\right\rfloor} O\left(\frac{1}{(\varepsilon n)^{h}} n^{h-1}\right)\right)=o\left(\mathscr{S}_{L} \rho^{n-m} \frac{(\log n)^{r-\hat{d}}}{n}\right) .
$$

From Theorem 3, we know $P_{L}(S, n) \sim\left(\frac{m}{n}\right)^{a-1} \mathscr{S}_{L} \rho^{n-m} \exp \left(-C_{L}(\rho ; J)\right)$. Using 10, we get,

$$
C_{L}\left(\rho ; J \cap\left(N_{n} \backslash N_{\left\lfloor(\log n)^{\log \log n\rfloor}\right.}\right)\right)=o(1),
$$

namely

$$
P_{L}(S, n) \exp \left(C_{L}\left(\rho ; J \cap N_{\left\lfloor(\log n)^{\log \log n}\right\rfloor}\right)\right) \sim \mathscr{S}_{L} \rho^{n-m} .
$$

We have

$$
\begin{aligned}
& \sum_{k=\lfloor\varepsilon n\rfloor}^{n-1} P\left(X_{n}^{[r]}(S)>k\right) \leq n P\left(X_{n}^{[r]}(S)>\lfloor\varepsilon n\rfloor\right) \\
& =o\left(P_{L}(S, n) \exp \left(C_{L}\left(\rho ; J \cap N_{\left\lfloor(\log n)^{\log \log n}\right\rfloor}\right)\right)(\log n)^{r-\hat{d}}\right) .
\end{aligned}
$$

This part is completed.

Finally, combining (18) with (11), 13) and (14), we finish our proof.

For some special restricted patterns, the above result can be simplified using Theorems 3 and 4 . 
Corollary 5 Suppose $r=O(1), a=1$ and $J=\emptyset$. Then, for the labeled case,

$$
E\left(X_{n}^{[r]}\right) \sim \frac{1}{r ! \exp (\gamma+K)}(\log n)^{r},
$$

and for the unlabeled case,

$$
E\left(X_{n}^{[r]}\right) \sim \frac{1}{\left.r ! \exp \left(\gamma+K+r_{0}\right)\right)}(\log n)^{r} .
$$

Proof: We can prove this corollary by applying Theorem 7 , Corollary 2 , and Corollary 4

\section{Examples.}

In this section, we provide several examples which cover some important decomposable combinatorial structures, such as permutations, children's yards, and polynomials over finite fields. These applications may be of interest for practitioners in cryptography; see (3) and (15).

Example 1: (Cycles in Permutations). Permutations are labeled structures which are decomposed into cycles. The component generating function is

$$
C(z)=\log \frac{1}{1-z},
$$

which is of logarithmic type with $\rho=a=1$. Suppose we want to know the probability that a random permutation of size $n$ has exactly 2 cycles of size 2,1 cycle of size 4 , and the size of its 10th smallest cycle is bigger than $\lfloor\log n\rfloor$. Applying Theorem 3 and Corollary 2 , with $S(2)=2$, $S(4)=1$, and $r=10$, we have

$$
P\left(X_{n}^{[10]}(S)>\lfloor\log n\rfloor\right) \sim \frac{(\log \log n)^{6}}{23040 e^{\gamma} \log n} .
$$

Applying Theorems 3 and 7, Corollary 2, and Lemma 1, we have

$$
\begin{aligned}
E\left(X_{n}^{[10]}(S)\right) & \sim \sum_{k=0}^{\left\lfloor\frac{n}{\log n \log \log n}\right\rfloor} P\left(X_{n}^{[10]}(S)>k\right) \\
& \sim \sum_{k=0}^{\lfloor\log n\rfloor} P\left(X_{n}^{[10]}(S)>k\right)+\sum_{k=\lfloor\log n\rfloor+1}^{\left\lfloor\frac{n}{\log n \log \log n}\right\rfloor} P\left(X_{n}^{[10]}(S)>k\right) \\
& \sim O(\log n)+\sum_{k=\lfloor\log n\rfloor+1}^{\left\lfloor\frac{n}{\log n \log \log n}\right\rfloor} P_{L}(S, n) \frac{\exp (3 / 4-\gamma)}{6 !} \frac{(\log k)^{6}}{k} \\
& \left.\sim O(\log n)+\sum_{k=\lfloor\log n\rfloor+1} \frac{e^{-\gamma}}{\log n \log \log n}\right\rfloor \\
& \sim O(\log n)+\frac{e^{-\gamma}}{161280}(\log n)^{7} \\
& \sim \frac{e^{-\gamma}}{161280}(\log n)^{7} .
\end{aligned}
$$


Example 2: (Children's Yards). Introduced by Stanley (16) in 1978, Children's yards are labeled structures made of rounds (directed cycles) with one child (node) in the center of each round. The component generating function is

$$
C(z)=z \log \frac{1}{1-z}=\log \frac{1}{1-z}+(z-1) \log \frac{1}{1-z}
$$

Since $(z-1) \log (1-z)^{-1} \rightarrow 0$ when $z \rightarrow 1, C(z)$ is of logarithmic type with $a=1$ and $\rho=1$. Let us estimate the probability that there is no cycle of size smaller than $\lfloor\log n\rfloor$ and the size of the $\lfloor\log \log n\rfloor$ th smallest cycle is bigger than $(\log n)^{\log \log n}$. According to Corollary 2 we get

$$
P\left(X_{n}^{[\lfloor\log \log n\rfloor]}(S)>(\log n)^{\log \log n}\right) \sim \frac{e^{-\gamma}(\log \log n)^{2\lfloor\log \log n\rfloor-2}}{(\log n)^{\log \log n(\lfloor\log \log n\rfloor-1) !}} .
$$

Example 3: (Irreducible Factors in Polynomials over Finite Fields). A typical unlabeled structure is the set of polynomials over finite fields. The generating function for polynomials over $\mathbb{F}_{q}$ is

$$
U(z)=\exp \left(C(z)+\frac{C\left(z^{2}\right)}{2}+\cdots\right)=\frac{1}{1-q z}, \quad \text { with } \quad C(z)=\log \frac{1}{1-q z}+F(z)
$$

where $F(z)$ is analytic in $|z|<q^{-1 / 2}$. Of course, there is a more precise expression for $C(z)$ but this form is enough for our interests here; see (3).

Let us consider, for example, the restricted pattern $S(2)=1$ and $S(\lfloor\log n\rfloor)=1$. Then, what is the probability that the 10th smallest irreducible factor has degree bigger than, say, $\sqrt{n}$ ? Applying Corollary 4, we have for each fixed $q$

$$
P\left(X_{n}^{[10]}(S)>\sqrt{n}\right) \sim \frac{\left(1-\frac{1}{q}\right)(\log n)^{6}}{2^{8} 7 ! \exp \left(\gamma+F(1 / q)+r_{0}\right) \sqrt{n}} .
$$

\section{Future Work}

In this paper, we study the size of the $r$ th smallest component for decomposable structures in the exp-log class with a given restricted pattern. We also provide the first moment for the size of the $r$ th smallest component. We now give some natural extensions of this work.

First, one could study higher moments for the size of the $r$ th smallest component in the exp-log class. Panario and Richmond (14) give the equivalent result for the simpler case of the empty pattern.

Furthermore, it seems plausible that the methods developed in this paper could allow the study of the $r$ th smallest component of decomposable structures whose component generating functions are of algebraic-logarithmic type.

Finally, the distribution of the size of the $r$ th largest component is another potential subject of study. In the largest component case, new methods will have to be provided. Indeed, the largest sizes among the components in the restricted pattern would exceed the range allowed in the theorems given in this paper. Gourdon's fundamental work (9) could be helpful in that study. 


\section{References}

[1] CAR,M. Théorèmes de densité dans $\mathbb{F}_{q}[x]$. Acta Arith. 48 (1987), 145-165.

[2] Dong,L., Gao,Z. and Panario,D. Enumeration of combinatorial decomposable structures with restricted patterns. Annals of Combinatorics, to appear.

[3] Flajolet,P., Gourdon,X. And Panario,D. The complete analysis of a polynomial factorization algorithm over finite fields. Journal of Algorithms, 40 (2001), 37-81.

[4] Flajolet,P. and Odlyzko,A. Singularity analysis of generating functions. SIAM J. Discrete Math. 3 (2) (1990), 216-240.

[5] Flajolet,P. And Sedgewick,R. Analytic Combinatorics, to appear.

[6] Flajolet,P. And Soria,M. Gaussian limiting distribution for the number of components in combinatorial structures. J. Combin. Theory Ser. A 53 (1990), 165-182.

[7] Goncharov,V. Sur la distribution des cycles dans une permutation.. Acad. Sci. URSS(N.S.) 35 (1942), 267-269.

[8] Goncharov,V. On the field of combinatorial analysis. Amer. Math. Soc. Transl. 2 (1962), $1-46$.

[9] Gourdon,X. Largest component in random combinatorial structures. Discrete Mathematics 180 (1998), 185-209.

[10] Knopfmacher,A. And Warlimont,R. Distinct degree factorizations for polynomials over a finite field. Trans. Amer. Math. Soc 347 (1995), 2235-2243.

[11] Knopfmacher,A. And Warlimont,R. Counting permutations and polynomials with a restricted factorization pattern. Australasian Journal of Combinatorics 13 (1996), 151-162.

[12] OdLyzko,A. Discrete logarithms in finite fields and their cryptographic significance. Lecture Notes in Computer Science 209 (1985), 224-314.

[13] Panario,D. And Richmond,B. Analysis of Ben-Or's polynomial irreducibility test. Random Struct. Algorithms 13 (1998), 439-456.

[14] Panario,D. And Richmond,B. Smallest components in decomposable structures: exp-log class. Algorithmica 29 (2001), 205-226.

[15] Quisquater,J.J. And Delescaille,J.P. How easy is collision search! Application to DES. Lecture Notes in Computer Science 434 (1989), 429-433.

[16] Stanley,R. Generating functions. In Studies in Combinatorics, G.-C. Rota, Ed., vol. 17 of MAA Studies in Mathematics, Mathematical Association of America, Washington, DC, 1978, pp. 100-141. 